\title{
Criminologie
}

\section{Le renouvellement des listes noires de la lutte contre l'argent sale}

\section{Gilles Favarel-Garrigues}

Volume 38, numéro 2, automne 2005

La police en pièces détachées

URI : https://id.erudit.org/iderudit/012663ar

DOI : https://doi.org/10.7202/012663ar

Aller au sommaire du numéro

\section{Éditeur(s)}

Les Presses de l'Université de Montréal

ISSN

0316-0041 (imprimé)

1492-1367 (numérique)

Découvrir la revue

Citer cet article

Favarel-Garrigues, G. (2005). Le renouvellement des listes noires de la lutte contre l'argent sale. Criminologie, 38(2), 91-102.

https://doi.org/10.7202/012663ar
Résumé de l'article

La lutte contre le financement du terrorisme constitue l'une des principales facettes de la politique menée en réponse aux événements du 11 septembre 2001. Le renforcement de ce combat s'est largement inspiré de la lutte antiblanchiment, telle qu'elle s'est organisée depuis 1989. Dans cet article, nous analysons comment s'articulent ces deux missions, notamment comment la mise en oeuvre de la lutte contre le financement du terrorisme modifie la pratique de l'antiblanchiment au sein des établissements financiers. Nous montrons que les acteurs financiers privés mènent une activité désormais plus personnalisée et plus dévouée à l'assistance aux enquêtes judiciaires. Les contraintes normatives associées à la lutte contre le financement du terrorisme conduisent à une professionnalisation des acteurs privés, mais leurs effets demeurent hypothétiques. 


\title{
Le renouvellement des listes noires de la lutte contre l'argent sale
}

\author{
Gilles Favarel-Garrigues * \\ CERI-Sciences Po (Centre d'études et de recherches internationales) \\ favarel@ceri-sciences-po.org
}

\begin{abstract}
RÉSUMÉ • La lutte contre le financement du terrorisme constitue l'une des principales facettes de la politique menée en réponse aux événements du 11 septembre 2001. Le renforcement de ce combat s'est largement inspiré de la lutte antiblanchiment, telle qu'elle s'est organisée depuis 1989. Dans cet article, nous analysons comment s'articulent ces deux missions, notamment comment la mise en œuvre de la lutte contre le financement du terrorisme modifie la pratique de l'antiblanchiment au sein des établissements financiers. Nous montrons que les acteurs financiers privés mènent une activité désormais plus personnalisée et plus dévouée à l'assistance aux enquêtes judiciaires. Les contraintes normatives associées à la lutte contre le financement du terrorisme conduisent à une professionnalisation des acteurs privés, mais leurs effets demeurent hypothétiques.
\end{abstract}

ABSTRACT - The struggle against the financing of terrorism is one of the main aspects of the war on terror that was launched after September 11 events of 2001. This struggle has been patterned after the strategy used against money laundering that was launched in 1989. In this paper, I examine how both strategies against the financing of terrorism and against money laundering by organized crime have been brought together, focusing on how much the move to curb the financing of terrorism has transformed anti-money laundering practices in the private financial sector. I show that in order to comply with the needs of hindering the financing of terrorism, the private sector's answers are now more sensitive to the individual case, more reactive and better coordinated to assist investigations by public police and magistrates. The regulatory constraints underpinning this new mission have led private financial actors to professionalize their practices in counterterrorism, but their true impact on terrorism still remains to be assessed.

* Gilles Favarel-Garrigues est chercheur CNRS au Centre d'études et de recherches internationales (CERI-Sciences Po), Paris. Ses travaux portent à la fois sur la gestion internationale des délinquances transnationales et sur la transformation des politiques pénales et des institutions policières en Russie. La liste de ses publications figure sur le site du CERI: $<$ www.ceri-sciences-po.org/cerifr/cherlist/favarel.htm>. 
La lutte contre le financement du terrorisme constitue l'une des principales facettes de l'action menée par les États-Unis, les pays occidentaux et de nombreuses organisations internationales en réponse aux événements du 11 septembre 2001. Le renforcement du combat contre les «finances terroristes» s'est largement inspiré de la lutte antiblanchiment, telle qu'elle a progressé depuis le sommet du G7 de l'Arche en 1989. Dans cet article, nous souhaitons analyser comment s'articulent ces deux missions, notamment comment le déploiement de la lutte contre le financement du terrorisme modifie la pratique de l'antiblanchiment au sein des établissements financiers.

\section{Le recyclage du dispositif institutionnel antiblanchiment}

L'idée d'inclure un volet financier dans la guerre antiterroriste s'inscrit dans un contexte socio-historique propice à sa promotion, en particulier aux États-Unis. Depuis quelques décennies déjà, la lutte contre les produits du crime (proceeds of crime) s'est imposée comme un élément nécessaire au démantèlement des organisations criminelles, notamment spécialisées dans le commerce des stupéfiants. Cette action visait simultanément deux objectifs en cherchant à confisquer les revenus illicitement perçus, mais aussi à priver ces organisations des moyens nécessaires à la réalisation d'autres infractions (Naylor, 2002). Pour des raisons diverses, la mise en œuvre de la lutte contre les produits du crime aux ÉtatsUnis a cependant rapidement conduit à la volonté d'internationaliser cette action, afin de faciliter la coopération internationale et d'harmoniser les règles nationales de contrôle des transactions financières (Helleiner, 1999). La mise au point d'un dispositif international de lutte antiblanchiment en 1989 a constitué l'un des principaux résultats de cette volonté. Les dirigeants politiques des pays membres du G7 ont alors commencé à se doter d'un dispositif institutionnel spécialisé et ont créé un secrétariat intergouvernemental, le Groupe d'action financière contre le blanchiment des capitaux (GAFI), chargé de concevoir des standards internationaux appropriés et d'évaluer les législations nationales. Durant la décennie 1990, le GAFI n'a eu de cesse de diffuser des normes, des institutions et des pratiques professionnelles vouées à la lutte contre l'argent sale, au moyen de techniques diverses. Après avoir favorisé les pressions exercées par les pairs au cours de campagnes 
d'évaluation mutuelle et la création de GAFI régionaux, le GAFI s'est lancé en 2000 dans la publication annuelle d'une liste des «pays et territoires non coopératifs», destinée à désigner et à discréditer («naming and shaming») les maillons faibles du front mondial contre l'argent sale (Sheptycki, 2000; Williams et Baudin O'Hayon, 2002; FavarelGarrigues, 2003a; Godefroy et Lascoumes, 2004).

Au lendemain des événements du 11 septembre 2001, le dispositif antiblanchiment est apparu, aux yeux des responsables occidentaux, comme un cadre d'action adapté à l'organisation de la lutte contre le financement du terrorisme, tant au niveau international que national. Cette association n'était pourtant pas tout à fait évidente: l'argent joue un rôle secondaire par rapport au don de sa propre vie qu'exigent de nombreux attentats, et nombre d'actes terroristes se fomentent à partir de moyens limités et souvent issus d'opérations licites. La lutte contre le financement du terrorisme conduit à s'intéresser à des pratiques telles que l'envoi informel de fonds, la bienfaisance et la donation individuelle qui jusque-là n'entraient d'aucune manière dans le champ de l'antiblanchiment. Il convient à ce titre de rappeler qu'au sein même du GAFI, nombre d'experts doutaient de la pertinence du rapprochement de ces deux questionnements jusqu'au début de l'année 2001 (GAFI XII, 2001:18 $)$.

Deux arguments principaux ont justifié le rapprochement opéré, selon ses promoteurs. Il existe, d'une part, des pratiques sécantes au sein des deux vastes ensembles que constituent les opérations de blanchiment et de financement du terrorisme. Les frontières entre la violence politique et la violence criminelle peuvent s'avérer ténues dans de nombreux contextes régionaux et nationaux. La mise en œuvre et la diffusion de la lutte antiblanchiment reposent, d'autre part, sur la mobilisation des acteurs financiers privés et sur l'institutionnalisation de leurs relations avec la police et la justice, au service d'une mission pénale. La lutte antiblanchiment constitue donc une ressource pour soutenir promptement la lutte contre le financement du terrorisme: dans les deux cas, l'organisation du dispositif de surveillance des flux financiers est similaire, en demandant aux acteurs privés de détecter les transactions suspectes et de communiquer toute information utile aux autorités gouvernementales

1. Les références aux rapports annuels d'activité du GAFI sont mentionnées, conformément aux usages, par des chiffres romains et leur date de publication. Voir: <http:// www1.oecd.org/fatf/FATDocs_en.htm\#Annual>. 
compétentes. L'existence d'un lien logique entre les deux enjeux se reflète dans les dispositions du USA Patriot Act qui étend les obligations déclaratives des professions financières au-delà des injonctions prévues dans le cadre de la lutte antiblanchiment.

La promotion internationale de la lutte contre le financement du terrorisme s'est appuyée sur l'action du GAFI. Réuni symboliquement à Washington DC à la fin du mois d'octobre 2001, le secrétariat intergouvernemental a décidé d'étendre son mandat à la lutte contre le financement du terrorisme et de réaliser sa nouvelle mission conformément aux procédures de diffusion des recommandations antiblanchiment. Il s'est d'abord agi de créer de nouveaux standards internationaux, en l'occurrence des recommandations spécialement adaptées aux objectifs de la lutte contre le financement du terrorisme. Comme dans le cas de la lutte antiblanchiment, ces recommandations demandent aux États d'ajouter une nouvelle incrimination au code pénal, de modifier la procédure pénale en instaurant ou en facilitant le recours au gel, à la saisie et à la confiscation des capitaux suspects, d'imposer des obligations déclaratives aux acteurs économiques et financiers privés et de renforcer la coopération internationale. La diffusion de ces nouveaux standards internationaux s'opère selon les méthodes d'évaluation usuelles au sein du GAFI, mais un changement important frappe tous les observateurs: loin de se renforcer, la procédure de publication d'une «liste noire» des juridictions non coopératives semble marginalisée, voire abandonnée. Cette décision a priori surprenante reflète la nouvelle configuration du dispositif institutionnel international antiblanchiment, notamment le rôle accru que jouent les institutions financières internationales en son sein depuis la fin 2001 (Favarel-Garrigues, 2003b).

\section{La montée en puissance des acteurs financiers}

L'engagement actif des institutions financières internationales, en particulier du Fonds monétaire international (FMI), dans la lutte contre l'argent sale représente l'un des principaux effets institutionnels des événements du 11 septembre 2001. Les budgets et les effectifs consacrés à cette mission au FMI et à la Banque mondiale ont considérablement augmenté depuis cette date. Le Fonds a, par ailleurs, intégré les 40 recommandations du GAFI dans ses programmes d'évaluation du secteur financier (Financial Sector Assessment Programs) et dans ses rapports sur 
l'observation des standards et des codes (Reports on the Observance of Standards and Codes). Cela signifie que l'examen des dispositifs nationaux antiblanchiment relève désormais des compétences des institutions financières internationales. Au cours du premier semestre 2002, ces dernières ont recherché avec le GAFI une méthodologie commune afin de conduire les évaluations. Cette nouvelle approche a été instaurée en novembre 2002, lors du lancement d'un programme pilote d'évaluation de la lutte antiblanchiment et de la lutte contre le financement du terrorisme, pérennisé en mars 2004. En outre, le FMI propose une assistance technique aux États désireux de renforcer leur dispositif de lutte contre l'argent sale. Cette nouvelle préoccupation figure enfin dans les évaluations des centres financiers offshore que mène le $\mathrm{FMI}^{2}$.

Cette activité ne surgit toutefois pas ex nibilo; elle s'inscrit dans le prolongement de l'intérêt croissant que le FMI et la Banque mondiale ont pu porter aux travaux du GAFI au cours des années 1990. L'intérêt de ces institutions pour la lutte antiblanchiment s'est affirmé au milieu de cette décennie (Quirk, 1996; Tanzi, 1996), mais les réticences demeuraient nombreuses: le blanchiment apparaissait certes comme une menace globale pour la stabilité financière internationale, mais les réponses envisagées étaient jugées contre-productives. Elles favorisaient en effet un interventionnisme étatique qui risquait de restreindre la liberté des mouvements de capitaux, alors que l'autocontrôle des marchés financiers représentait le meilleur moyen de se protéger contre l'argent sale ${ }^{3}$. L'engagement des deux institutions financières internationales s'est renforcé à la fin des années 1990 lorsqu'elles ont décidé de dépêcher des observateurs lors des réunions plénières du GAFI. Leur présence renforçait d'ailleurs la pression exercée sur les «pays et territoires non coopératifs», enjoints d'adopter les standards internationaux. Enfin, c'est avant les événements de septembre 2001, plus exactement au mois d'avril, que les institutions financières internationales ont reconnu dans les 40 recommandations du GAFI, les normes appropriées pour lutter contre le blanchiment et se sont engagées à les intégrer dans leur activité d'évaluation.

Par ailleurs, la contribution accrue du FMI et de la Banque mondiale fait écho au rôle primordial que jouent les administrations financières

2. Pour une présentation synthétique de l'activité du FMI dans ce domaine, voir: $<$ http://www.imf.org/external/np/exr/facts/aml.htm>.

3. Voir, par exemple, les propos de l'ancien directeur général du FMI, M. Camdessus, lors d'une réunion du GAFI, in: GAFI IX, 1998, p. 41-42. 
dans le dispositif institutionnel antiblanchiment depuis sa création. Sur le plan national, la lutte contre l'argent sale justifie la coopération de plusieurs administrations, mais le ministère des Finances joue presque toujours un rôle prédominant. C'est généralement sous son autorité que travaille l'unité de renseignements financiers, chargée de collecter les déclarations de soupçons en provenance des établissements financiers privés et de les transmettre aux autorités judiciaires compétentes. En outre, les principaux représentants des États membres lors des réunions $\mathrm{du}$ GAFI proviennent des administrations financières nationales.

Le renforcement de l'engagement des institutions financières internationales dans la lutte contre l'argent sale modifie les modes de diffusion des standards internationaux dans ce domaine. D'un côté, la légitimité de ces institutions est supérieure à celle du GAFI. Alors que ce dernier compte aujourd'hui 33 membres, le Fonds et la Banque bénéficient d'une présence quasi universelle. Il est du coup moins évident d'associer la lutte antiblanchiment à une obsession des pays membres du G7, comme de nombreux pays extérieurs à cette enceinte ont pu l'exprimer au cours des années 1990, notamment lors des discussions à l'ONU sur la Convention internationale de lutte contre la criminalité organisée transnationale, finalement adoptée en décembre $2000^{4}$. D'un autre côté, l'engagement des institutions financières internationales a conduit le GAFI à renoncer à actualiser la liste des pays et territoires non coopératifs, soit l'un de ses principaux moyens de pression. Une telle liste heurte les sacro-saints principes d'uniformité, de volontariat et de coopération qui fondent l'activité du FMI et de la Banque mondiale. Au sein de ces institutions, l'évaluation ne s'impose pas, mais répond à la demande des États; ces derniers participent aux procédures mises en application et peuvent, au moins théoriquement, s'opposer à la publication des résultats.

La question du rôle du GAFI se pose du coup avec acuité. Le renouvellement du mandat de cette instance en 2004 pour huit ans a fourni l'occasion de s'interroger sur son avenir. Sa fonction principale est désormais d'édicter des normes internationales («standard setting»). Pour l'heure, le GAFI continue d'évaluer les pays membres, en promouvant une nouvelle série d'examens de leurs dispositifs nationaux, plus sensibles à l'application pratique de la législation antiblanchiment, mais

4. Malgré la volonté des États membres du G7, le texte de la Convention réfere aux standards internationaux de la lutte antiblanchiment, mais ne mentionne pas explicitement les recommandations du GAFI. 
le FMI et la Banque mondiale ont d'ores et déjà conduit cet exercice dans quelques cas (GAFI XVI, 2005 : 11). Pour ce qui est des pays nonmembres, les institutions financières internationales semblent s'imposer. Dans ce domaine, le GAFI se contentera désormais, selon sa propre terminologie, de «garantir que des mesures seront prises au niveau mondial pour combattre le blanchiment et le financement du terrorisme, notamment renforcer la coopération avec le FMI et la Banque mondiale» (GAFI XV, 2004: 8). La pérennisation du programme pilote des institutions financières internationales en mars 2004, puis la mise au point relative aux compétences respectives du Fonds, de la Banque, du GAFI et des GAFI régionaux dans l'évaluation des dispositifs nationaux antiblanchiment ${ }^{5}$ confirment ce constat. Les autres missions correspondent à l'activité habituelle du GAFI : étude des pratiques et réalisation de typologies du blanchiment, accueil de nouveaux membres et coordination de l'action des GAFI régionaux. La référence à la liste des «pays et territoires non coopératifs » est plus discrète que dans le passé (GAFI XVI, 2005 : 7). Le GAFI a certes renoncé à l'actualiser depuis 2002 et s'est employé à réduire le nombre d'inscrits, mais trois irréductibles (Myanmar, Nauru et Nigeria) figurent encore en février 2005 sur une liste qui, de toute évidence, ne constitue plus le moyen de pression qu'elle représentait quelques années auparavant.

La prédominance des acteurs financiers dans la lutte contre l'argent sale se reflète enfin dans la mise au point d'une ingénierie privée spécialisée depuis le 11 septembre 2001, destinée à aider les établissements financiers à réaliser leurs obligations face à la menace terroriste. Ce secteur d'activité en plein essor comprend l'offre de prestations (conseil, analyse juridique, formation du personnel, etc.) et d'outils informatiques spécialisés dans la prévention du blanchiment et du financement du terrorisme. Il existait certes avant 2001, mais s'est renouvelé en ajoutant à l'arsenal traditionnel de détection des transactions suspectes des listes destinées à repérer les clients suspects. La demande des établissements financiers est motivée par la volonté de réduire les risques qui pèsent sur leur activité, en renforçant les procédures liées à une meilleure «connaissance» de leurs clients (Know Your Customer ou KYC). L'investissement croissant des grands groupes internationaux de conseil dans l'assistance aux établissements financiers pourrait conduire à formuler l'hypothèse de

5. Voir le document d'août 2004 in: <http://www1.worldbank.org/finance/html/ amlcft/docs/FSAP_\&_OFC.pdf>. 
l'émergence d'une gouvernance globale de l'argent sale, dans laquelle les normes édictées par des instances intergouvernementales sont désormais davantage appliquées par des acteurs transnationaux privés.

\section{Vers une transformation du rôle des acteurs privés dans la lutte contre l'argent sale?}

L'émergence d'une ingénierie professionnelle spécialisée dans la détection des transactions suspectes conduit à une réflexion plus générale sur la pratique de la lutte antiblanchiment. En quoi la volonté de lutter contre le financement du terrorisme transforme-t-elle l'action menée contre l'argent sale? Compte tenu du caractère indétectable de certaines formes de financement du terrorisme, la lutte menée à son encontre à l'échelle internationale vise avant tout à faciliter, dans la pratique, la coopération judiciaire intergouvernementale. Les opérations internationales menées depuis le 11 septembre 2001 dans ce domaine cherchent à saisir les capitaux qu'une personne physique ou morale détient hors des frontières de la juridiction qui la suspecte. En conséquence, les impératifs de la lutte contre le terrorisme orientent l'action que mènent les acteurs financiers privés vers la neutralisation de cibles clairement identifiées. En témoigne la multiplication de listes de personnes physiques ou morales recherchées pour leur participation à des attentats ou étiquetées comme organisations terroristes. Enclenchée par les États-Unis ainsi que par des organisations internationales (ONU) et régionales (Union européenne), la constitution de telles listes s'observe également dans de nombreux pays, à l'instar de la Russie où elles fondent l'effort mené contre les finances terroristes ${ }^{6}$. En outre, les établissements financiers doivent désormais tenir compte des risques liés aux «personnes politiquement exposées », c'est-à-dire d'une liste étendue de hauts responsables nationaux et de leur entourage, comme le souligne, par exemple, le texte de la troisième directive européenne sur la lutte contre le blanchiment, récemment adoptée. La lutte contre l'argent sale se fait désormais à partir de nouvelles «listes noires» qui ne désignent plus

6. La présentation du cas russe pourrait, par ailleurs, conduire à d'intéressants développements sur la transformation de la lutte contre l'argent sale depuis le 11 septembre 2001. Rappelons, en effet, que ce pays figurait sur la liste noire du GAFI en 2000 et 2001, avant d'en être retiré l'année suivante, puis de devenir membre à part entière du secrétariat intergouvernemental à partir de 2003. Nous avons examiné les raisons de ce fulgurant revirement in: Favarel-Garrigues, 2003c. 
les juridictions «non coopératives», mais les opérateurs illégitimes au sein des circuits financiers internationaux, qu'il s'agisse de personnes physiques ou morales.

La personnalisation de l'action menée depuis le 11 septembre 2001 modifie les pratiques bancaires de lutte antiblanchiment qui, jusqu'à cette date, se consacraient prioritairement à la détection des transactions suspectes. Il serait certes faux de considérer qu'au cours des années 1990 l'application des normes du GAFI visait l'ensemble des pratiques de blanchiment. La définition de l'argent sale a toujours constitué le produit d'un consensus minimal entre les diverses professions engagées dans ce combat (acteurs policiers, judiciaires, financiers publics ou privés). C'est au nom de la lutte contre des cibles unanimement illégitimes, (trafic de stupéfiants, puis crime organisé transnational, puis financement du terrorisme), que la lutte antiblanchiment s'est progressivement imposée dans le monde. Comme il a été maintes fois montré, le blanchiment des capitaux issus d'infractions fiscales ou de formes de délinquance d'affaires commises par des acteurs plus légitimes n'a jamais suscité la même attention que les pratiques employées par les organisations criminelles afin de légaliser les produits de leurs méfaits (Godefroy et Lascoumes, 2004; Helleiner, 1999; Strange, 1998). L'action menée contre l'argent sale visait donc moins à réguler l'ensemble des flux transnationaux de capitaux d'origine illicite qu'à en réprimer certaines formes et à exclure des circuits financiers internationaux des acteurs jugés illégitimes par l'ensemble des professions participant au combat, du fait de leurs liens supposés avec des organisations criminelles ou des réseaux terroristes. Toutefois, jusqu'en 2001, cette action ne s'appuyait guère sur la détention, au sein des établissements financiers, de listes nominales de personnes physiques ou morales suspectes, mais sur des modalités techniques de détection impersonnelle des transactions suspectes. Dans la mesure où l'on considérait que la nécessité de dissimuler l'origine illicite de capitaux pouvait conduire les criminels à des opérations financières particulières et aisément identifiables, le caractère suspect de la transaction était davantage lié à ses propres caractéristiques qu'à l'identité supposée de l'opérateur ou du bénéficiaire.

Comment interpréter les effets d'un tel changement? Une solution simple serait de souligner les dangers qu'une telle approche comporte pour les libertés publiques, compte tenu des interrogations qui pèsent sur le mode de constitution des listes de personnes qualifiées de terroristes, selon une terminologie aux contours notoirement imprécis. Mais 
il ne faudrait pas pour autant se contenter de brandir l'épouvantail orwellien, car cette approche pourrait tout aussi bien s'avérer inutile dans un monde où les personnes physiques et morales peuvent aisément changer d'identité et où, répétons-le, la détention de moyens financiers ne semble pas toujours nécessaire pour commettre un attentat. À nos yeux, ce changement transforme avant tout le rôle des acteurs privés. Alors que, jusqu'à présent, leur engagement dans la prévention du blanchiment s'opérait de manière proactive, elle semble aujourd'hui plus réactive et davantage orientée vers l'assistance aux enquêtes judiciaires. L'internationalisation de la lutte contre le financement du terrorisme contribue à accélérer la diffusion des procédures de gel, de saisie et de confiscation de capitaux suspects sur l'ensemble de la planète, et ainsi à faciliter des enquêtes judiciaires contraintes par les frontières des juridictions nationales (Realuyo, 2004). Il est, de ce point de vue, révélateur que l'administration états-unienne étaie son appréciation des résultats de la lutte contre le financement du terrorisme en indiquant le nombre de personnes physiques et morales figurant sur les listes de terroristes et le montant des avoirs gelés au niveau international (Zarate, 2004). Enfin, le renforcement des obligations légales pesant sur le secteur financier privé a accéléré le processus de professionnalisation du personnel en charge de la détection des transactions suspectes, comme le soulignent, dans un pays comme la France depuis le début des années 2000, les besoins de recrutement des établissements bancaires et des compagnies d'assurance, la constitution de forums spécialisés et l'émergence de formations universitaires dédiées à la lutte contre le blanchiment et la délinquance financière.

L'avenir dira si le renforcement de la lutte contre le financement du terrorisme marginalisera ou non l'antiblanchiment. Les observateurs semblent partagés sur ce point : certains considèrent que la lutte contre le financement du terrorisme sert l'antiblanchiment, dans la mesure où elle accroît la collaboration des acteurs financiers privés dans les procédures judiciaires, tandis que d'autres estiment que cette nouvelle priorité décrédibilise une décennie de sensibilisation à l'argent sale. La volonté d'intensifier la lutte contre le financement du terrorisme a pour l'heure conduit à une reconfiguration du dispositif institutionnel international de lutte contre l'argent sale, en redéfinissant notamment les missions du GAFI et en conférant au FMI et à la Banque mondiale un rôle désormais déterminant. L'engagement de ces institutions, notamment du Fonds, dans le combat est manifeste, même si des interrogations demeurent 
quant à leur compétence pour évaluer les facettes policières et judiciaires de l'action menée au niveau national, et quant à l'importance de ce dossier dans l'octroi de prêts. L'organisation de la lutte contre le financement du terrorisme semble avant tout viser à renforcer la coopération judicaire internationale et à homogénéiser les procédures pénales. Ce faisant, elle réoriente l'activité des acteurs financiers privés: tenus au cours des années 1990 de détecter et de signaler les transactions anormales, il leur est désormais demandé de surveiller les opérations liées à des personnes physiques ou morales suspectes. Le renforcement de leurs obligations légales les conduit à rendre des comptes, notamment en réorganisant leurs services, en recrutant des agents spécialisés, en formant l'ensemble de leur personnel et en s'équipant d'outils techniques coûteux proposés par des prestataires évoluant dans un secteur d'activité en plein essor. Les effets de cet investissement massif demeurent toutefois hypothétiques: s'ils prémunissent les établissements financiers des reproches des autorités judiciaires sur leur éventuel laxisme, ils n'attestent nullement l'efficacité, voire la pertinence, du volet financier de la guerre contre le terrorisme.

\section{Références}

Favarel-Garrigues, G. (2003a). «Crime organisé transnational» et lutte antiblanchiment. In J. Laroche (ed.), Mondialisation et gouvernance mondiale (161-174). Paris : IRIS et PUF.

Favarel-Garrigues, G. (2003b). L'évolution de la lutte antiblanchiment depuis le 11 septembre 2001. Critique internationale, 20, juillet, 37-47.

Favarel-Garrigues, G. (2003c). La création d'un dispositif antiblanchiment en Russie. In G. Favarel-Garrigues (ed.), Criminalité, police et gouvernement: trajectoires post-communistes (135-161). Paris : L'Harmattan.

Godefroy, T. \& Lascoumes, P. (2004). Le capitalisme clandestin. Paris : La découverte. Helleiner, E. (1999). State Power and the Regulation of Illicit Activity in Global Finance. In H. R. Friman, P. Andreas (ed), The Illicit Global Economy and State Power (53-90). Lanham: Rowman \& Littlefield.

Naylor, R. T. (2002). Wages of Crime. Black Markets, Illegal Finance and the Underworld Economy. Ithaca: Cornell University Press.

Quirk, P. (1996). Macroeconomic Implications of Money Laundering. Washington DC : International Monetary Fund.

Realuyo, C. (2004). La mise en place de moyens de lutte contre le financement du terrorisme. Perspectives économiques, septembre, 11-15.

Sheptycki, J. (2000). Policing the Virtual Launderette. Money Laundering and Global Governance. In J. Sheptycki (ed.), Issues in Transnational Policing (154160). London: Routledge. 
Strange, S. (1998). Mad Money. Manchester : Manchester University Press.

Tanzi, V. (1996). Money Laundering and the International Financial System. Washington D.C: International Monetary Fund

Williams, P. \& Baudin-O’Hayon, G. (2002). Global Governance, Transnational Organized Crime and Money Laundering. In D. Held, A. McGrew (eds), Governing Globalization. Power, Authority and Global Governance (127-144). Cambridge: Polity Press.

Zarate, J. C. (2004). Réduire les terroristes à la faillite. Perspectives économiques, septembre, 3-6. 